\title{
On $\mathscr{I}_{\sigma}$-convergence of folner sequence on amenable semigroups
}

\author{
Omer Kisi ${ }^{1}$ and Burak Cakal ${ }^{2}$ \\ ${ }^{1}$ Deparment of Mathematics, Faculty of Science, Bartın University, Bartın, Turkey \\ ${ }^{2}$ Deparment of Mathematics, Bartın University, Bartın, Turkey
}

Received: 9 April 2018, Accepted: 23 May 2018

Published online: 4 July 2018.

\begin{abstract}
In this paper, the concepts of $\sigma$-uniform density of subsets $A$ of the set $\mathbb{N}$ of positive integers and corresponding $\mathscr{I}_{\sigma^{-}}$ convergence of functions defined on discrete countable amenable semigroups were introduced. Furthermore, for any Folner sequence inclusion relations between $\mathscr{I}_{\sigma}$-convergence and invariant convergence also $\mathscr{I}_{\sigma}$-convergence and $\left[V_{\sigma}\right]_{p}$-convergence were given. We introduce the concept of $\mathscr{I}_{\sigma}$-statistical convergence and $\mathscr{I}_{\sigma}$-lacunary statistical convergence of functions defined on discrete countable amenable semigroups. In addition to these definitions, we give some inclusion theorems. Also, we make a new approach to the notions of $[V, \lambda]$-summability, $\sigma$-convergence and $\lambda$-statistical convergence of Folner sequences by using ideals and introduce new notions, namely, $\mathscr{I}_{\sigma-}[V, \lambda]$-summability, $\mathscr{I}_{\sigma}-\lambda$-statistical convergence of Folner sequences. We mainly examine the relation between these two methods as also the relation between $\mathscr{I}_{\sigma}$-statistical convergence and $\mathscr{I}_{\sigma}$ - $\lambda$-statistical convergence of Folner sequences introduced by the author recently.
\end{abstract}

Keywords: Folner sequence, amenable group, inferior, superior, $\mathscr{I}$-convergence.

\section{Introduction}

Statistical convergence of sequences of points was introduced by Fast [5]. Schoenberg [27] established some basic properties of statistical convergence and also studied the concept as a summability method.

The natural density of a set $K$ of positive integers is defined by

$$
\delta(K):=\lim _{n \rightarrow \infty} \frac{1}{n}|\{k \leq n: k \in K\}|,
$$

where $|k \leq n: k \in K|$ denotes the number of elements of $K$ not exceeding $n$.

A number sequence $x=\left(x_{k}\right)$ is said to be statistically convergent to the number $L$ if for every $\varepsilon>0$,

$$
\lim _{n \rightarrow \infty} \frac{1}{n}\left|\left\{k \leq n:\left|x_{k}-L\right| \geq \varepsilon\right\}\right|=0
$$

In this case we write $s t-\lim x_{k}=L$. Statistical convergence is a natural generalization of ordinary convergence. If $\lim x_{k}=L$, then $s t-\lim x_{k}=L$. The converse does not hold in general.

By a lacunary sequence we mean an increasing integer sequence $\theta=\left\{k_{r}\right\}$ such that $k_{0}=0$ and $h_{r}=k_{r}-k_{r-1} \rightarrow \infty$ as $r \rightarrow \infty$. Throughout this paper the intervals determined by $\theta$ will be denoted by $I_{r}=\left(k_{r-1}, k_{r}\right]$.

*Corresponding author e-mail: okisi@bartin.edu.tr and burakcakal@gmail.com 
The concept of lacunary statistical convergence was defined by Fridy and Orhan [6]. Also, Fridy and Orhan gave the relationships between the lacunary statistical convergence and the Cesàro summability.

A sequence $x=\left(x_{k}\right)$ is said to be lacunary statistically convergent to the number $L$ if for every $\varepsilon>0$ the set

$$
K_{\varepsilon}=\left\{k \in \mathbb{N}:\left|x_{k}-L\right| \geq \varepsilon\right\}
$$

has lacunary density zero, i.e. $\delta_{\theta}\left(K_{\varepsilon}\right)=0$. In this case we write $S_{\theta}-\lim x_{k}=L$ or $x_{k} \rightarrow L\left(S_{\theta}\right)$. That is,

$$
\lim _{r \rightarrow \infty} \frac{1}{h_{r}}\left|\left\{k \in I_{r}:\left|x_{k}-L\right| \geq \varepsilon\right\}\right|=0 .
$$

Let $\sigma$ be a one-to-one mapping of the set of positive integers into itself such that $\sigma^{m}(n)=\left(\sigma^{m-1}(n)\right), m=1,2,3, \ldots . \mathrm{A}$ continuous linear functional $\Phi$ on $l_{\infty}$, the space of real bounded sequences, is said to be an invariant mean or a $\sigma$ mean, if and only if,

(1) $\Phi(x) \geq 0$, for all sequences $x=\left(x_{n}\right)$ with $x_{n} \geq 0$ for all $n$;

(2) $\Phi(e)=1$, where $e=(1,1,1, \ldots)$;

(3) $\Phi\left(x_{\sigma(n)}\right)=\Phi(x)$ for all $x \in l_{\infty}$.

The mapping $\Phi$ are assumed to be one-to-one such that $\sigma^{m}(n) \neq n$ for all positive integers $n$ and $m$, where $\sigma^{m}(n)$ denotes the $m$ th iterate of the mapping $\sigma$ at $n$. Thus, $\Phi$ extends the limit functional on $c$, the space of convergent sequences, in the sense that $\Phi(x)=\lim x$, for all $x \in c$. In case $\sigma$ is translation mapping $\sigma(n)=n+1$, the $\sigma$ mean is often called a Banach limit and $V_{\sigma}$, the set of bounded sequences all of whose invariant means are equal, is the set of almost convergent sequences.

It can be shown that

$$
V_{\sigma}=\left\{x=\left(x_{n}\right): \lim _{n} t_{m n}(x)=L, \text { uniformly in } m, L=\sigma-\lim x\right\}
$$

where

$$
t_{m n}(x)=\frac{x_{m}+x_{\sigma(m)}+x_{\sigma^{2}(m)}+\ldots+x_{\sigma^{n}(m)}}{n+1}
$$

The concept of strongly $\sigma$-convergence was defined by Mursaleen in [18].

A bounded sequence $x=\left(x_{k}\right)$ is said to be strongly $\sigma$-convergent to $L$ if

$$
\lim _{m \rightarrow \infty} \frac{1}{m} \sum_{k=1}^{\infty}\left|x_{\sigma^{k}(n)}-L\right|=0
$$

uniformly in $n$. In this case we will write $x_{k} \rightarrow L\left[V_{\sigma}\right]$.

Savaş and Nuray [22] introduced the concepts of $\sigma$-statistically convergence and lacunary $\sigma$-statistically convergence and gave some inclusion relations.

A family of sets $\mathscr{I} \subseteq 2^{\mathbb{N}}$ is called an ideal if and only if

(1) $\emptyset \in \mathscr{I}$,

(2) For each $A, B \in \mathscr{I}$ we have $A \cup B \in \mathscr{I}$,

(3) For each $A \in \mathscr{I}$ and each $B \subseteq A$ wehave $B \in \mathscr{I}$. 
An ideal is called non-trivial if $\mathbb{N} \notin \mathscr{I}$ and non-trivial ideal is called admissible if $\{n\} \in \mathscr{I}$ for each $n \in \mathbb{N}$. A family of sets $\mathscr{F} \subseteq 2^{\mathbb{N}}$ is a filter if and only if

(i) $\emptyset \notin \mathscr{F}$,

(ii) For each $A, B \in \mathscr{F}$ we have $A \cap B \in \mathscr{F}$,

(iii) For each $A \in \mathscr{F}$ and each $B \supseteq A$ we have $B \in \mathscr{F}$.

If $\mathscr{I}$ is proper ideal of $\mathbb{N}$ (i.e., $\mathbb{N} \notin \mathscr{I}$ ), then the family of sets

$$
\mathscr{F}(\mathscr{I})=\{M \subset \mathbb{N}: \exists A \in \mathscr{I}: M=\mathbb{N} \backslash A\}
$$

is a filter of $\mathbb{N}$ it is called the filter associated with the ideal. Filter is a dual notion of ideal.

The notion of ideal convergence was introduced first by Kostyrko et al. [10] as a generalization of statistical convergence $[11,5]$. More applications of ideals can be found in $[12,13]$.

In another direction the idea of $\lambda$-statistical convergence was introduced and studied by Mursaleen [17] as an extension of the $[V, \lambda]$ summability of [14].

Let $\lambda=\left(\lambda_{n}\right)$ is a non-decreasing sequence of positive numbers tending to $\infty$ such that $\lambda_{n+1} \leq \lambda_{n}+1, \lambda_{1}=1$. The generalized de la Valee-Pousin meaan is defined by

$$
t_{n}(x)=\frac{1}{\lambda_{n}} \sum_{k \in I_{n}} x_{k}
$$

where $I_{n}=\left[n-\lambda_{n}+1, n\right]$.

A sequence $x=\left(x_{k}\right)$ is said to be $(V, \lambda)$-summable to a number $L$ if

$$
\lim _{n \rightarrow \infty} t_{n}(x)=L
$$

If $\lambda_{n}=n$, then $(V, \lambda)$-summability reduces to $(C, 1)$-summability. We write

$$
[C, 1]=\left\{x=\left(x_{n}\right): \exists L \in \mathbb{R}, \lim _{n \rightarrow \infty} \frac{1}{n} \sum_{k=1}^{n}\left|x_{k}-L\right|=0\right\}
$$

and

$$
[V, \lambda]=\left\{x=\left(x_{n}\right): \exists L \in \mathbb{R}, \lim _{n \rightarrow \infty} \frac{1}{\lambda_{n}} \sum_{k \in I_{n}}\left|x_{k}-L\right|=0\right\}
$$

for the sets of sequences $x=\left(x_{k}\right)$ which are strongly Cesaro summable and strongly $(V, \lambda)-$ summable to $L$, i.e. $x_{k} \rightarrow L$ $[C, 1]$ and $x_{k} \rightarrow L[V, \lambda]$ respectively. He denoted $\Lambda$, the set of all non-decreasing sequences $\lambda=\left(\lambda_{n}\right)$ of positive numbers tending to $\infty$ such that $\lambda_{n+1} \leq \lambda_{n}+1$ and $\lambda_{1}=1$.

In [23], the concepts of $\sigma$-uniform density of subsets $A$ of the set $\mathbb{N}$ of positive integers and corresponding $\mathscr{I}_{\sigma}$-convergence were introduced. Also, inclusion relations between $\mathscr{I}_{\sigma}$-convergence and invariant convergence also $\mathscr{I}_{\sigma}$-convergence and $\left[V_{\sigma}\right]_{p}$. 
Let $A \subseteq \mathbb{N}$ and

$$
\begin{aligned}
s_{m} & :=\min _{n}\left|A \cap\left\{\sigma(n), \sigma^{2}(n), \ldots, \sigma^{m}(n)\right\}\right| \\
S_{m} & :=\max _{n}\left|A \cap\left\{\sigma(n), \sigma^{2}(n), \ldots, \sigma^{m}(n)\right\}\right| .
\end{aligned}
$$

If the following limits exist

$$
\underline{V}(A):=\lim _{m \rightarrow \infty} \frac{s_{m}}{m}, \bar{V}(A):=\lim _{m \rightarrow \infty} \frac{S_{m}}{m}
$$

then they are called a lower and an upper $\sigma$-uniform density of the set $A$, respectively.If $V(A)=\underline{V}(A)=\bar{V}(A)$ is called the $\sigma$-uniform density of $A$.

Denote by $\mathscr{I}_{\sigma}$ the class of all $A \subseteq \mathbb{N}$ with $V(A)=0$.

A sequence $x=\left(x_{n}\right)$ is said to be $\mathscr{I}_{\sigma}$-convergent to the number $L$ if for every $\varepsilon>0$

$$
A(\varepsilon):=\left\{k:\left|x_{k}-L\right| \geq \varepsilon\right\}
$$

belongs to $\mathscr{I}_{\sigma} ;$ i.e., $V\left(A_{\varepsilon}\right)=0$. In this case we write $\mathscr{I}_{\sigma}-\lim x_{k}=L$.

In [21], they made a new approach to the notions of $[V, \lambda]$-summability and $\lambda$-statistical convergence by using ideals and introduce new notions, namely, $\mathscr{I}$ - $[V, \lambda]$ - summability and $\mathscr{I}$ - $\lambda$-statistical convergence. They mainly examined the relation between these two new methods as also the relation between $\mathscr{I}$ - $\lambda$-statistical convergence and $\mathscr{I}$-statistical convergence introduced by the authors recently.

Recently, Das, Savas and Ghosal [2] introduced new notions, namely $\mathscr{I}$-statistical convergence and $\mathscr{I}$-lacunary statistical convergence by using ideal, convergence, investigated their relationship, and made some observations about these classes.

Let $\mathrm{G}$ be a discrete countable amenable semigroup with identity in which both right and left cancelation laws hold, and $w(G)$ and $m(G)$ denote the spaces of all real valued functions and all bounded real functions $f$ on $G$ respectively. $m(G)$ is a Banach space with the supremum norm $\|f\|_{\infty}=\sup \{|f(g)|: g \in G\}$. Nomika [26] showed that, if $G$ is countable amenable group, there exists a sequence $\left\{S_{n}\right\}$ of finite subsets of $G$ such that $(i) G=\cup_{i=1}^{\infty} S_{n}$, (ii) $S_{n} \subset S_{n+1}$, $n=1,2,3, \ldots$, (iii) $\lim _{n \rightarrow \infty} \frac{\left|S_{n} g-\cap S_{n}\right|}{\left|S_{n}\right|}=1, \lim _{n \rightarrow \infty} \frac{\left|g S_{n}-\cap S_{n}\right|}{\left|S_{n}\right|}=1$ for all $g \in G$. Here $|A|$ denotes the number of elements in the finite set $A$. Any sequence of finite subsets of $G$ satisfying $(i),(i i)$ and (iii) is called a Folner sequence for $G$.

The sequence $S_{n}=\{0,1,2, \ldots, n-1\}$ is a familiar Folner sequence giving rise to the classical Cesàro method of summability.

Amenable semigroups were studied by [1]. The concept of summability in amenable semigroups was introduced in [15], [16]. In [3], Douglas extended the notion of arithmetic mean to amenable semigroups and obtained a characterization for almost convergence in amenable semigroups.

In [25], the notions of convergence and statistical convergence, statistical limit point and statistical cluster point to functions on discrete countable amenable semigroups were introduced.

The purpose of the study [28] was to extend the notions of $\mathscr{I}$-convergence, $\mathscr{I}$-limit superior and $\mathscr{I}$-limit inferior, $\mathscr{I}$-cluster point and $\mathscr{I}$-limit point to functions defined on discrete countable amenable semigroups. Also, he made a new 
approach to the notions of $[V, \lambda]$-summability and $\lambda$-statistical convergence by using ideals and introduced new notions, namely, $\mathscr{I}$-[V, $\lambda]$-summability and $\mathscr{I}$ - $\lambda$-statistical convergence to functions defined on discrete countable amenable semigroups.

\section{Definitions and notations}

Definition 1. [23] Let $G$ be a discrete countable amenable semigroup with identity in which both right and left cancelation laws hold. $f \in w(G)$ is said to be convergent to $s$, for any Folner sequence $\left\{S_{n}\right\}$ for $G$, if for each $\varepsilon>0$ there exists $k_{0} \in \mathbb{N}$ such that $|f(g)-s|<\varepsilon$ for all $m>k_{0}$ and $g \in G \backslash S_{m}$.

Definition 2. [23] Let $G$ be a discrete countable amenable semigroup with identity in which both right and left cancelation laws hold. $f \in w(G)$ is said to be a Cauchy sequence for any Folner sequence $\left\{S_{n}\right\}$ for $G$, if for each $\varepsilon>0$ there exists $k_{0} \in \mathbb{N}$ such that $|f(g)-f(h)|<\varepsilon$ for all $m>k_{0}$ and $g \in G \backslash S_{m}$.

Definition 3. [23] Let $G$ be a discrete countable amenable semigroup with identity in which both right and left cancelation laws hold. $f \in w(G)$ is said to be strongly summable to s, for any Folner sequence $\left\{S_{n}\right\}$ for $G$, if

$$
\lim _{n \rightarrow \infty} \frac{1}{\left|S_{n}\right|} \sum_{g \in S_{n}}|f(g)-s|=0,
$$

where $\left|S_{n}\right|$ denotes the cardinality of the set $S_{n}$.

The upper and lower Folner densities of a a set $S \subset G$ are defined by

$$
\bar{\delta}(S)=\lim _{n \rightarrow \infty} \sup \frac{1}{\left|S_{n}\right|}\left|\left\{g \in S_{n}: g \in S\right\}\right|
$$

and

$$
\underline{\delta}(S)=\lim _{n \rightarrow \infty} \inf \frac{1}{\left|S_{n}\right|}\left|\left\{g \in S_{n}: g \in S\right\}\right|
$$

respectively $\bar{\delta}(S)=\underline{\delta}(S)$, then

$$
\delta(S)=\lim _{n \rightarrow \infty} \frac{1}{\left|S_{n}\right|}\left|\left\{g \in S_{n}: g \in S\right\}\right|
$$

is called Folner density of $S$. Take $G=\mathbb{N}, S_{n}=\{0,1,2, \ldots, n-1\}$ and $S$ be the set of positive integers with leading digit 1 in the decimal expansion. The set $S$ has no Folner density with respect to the Folner sequence $\left\{S_{n}\right\}$, since $\underline{\delta}(S)=\frac{1}{9}$, $\bar{\delta}(S)=\frac{5}{9}$. To facililate this idea we introduce the following notion: If $f$ is function such that $f(g)$ satisfies property $P$ for all $g$ expect a set of Folner density zero, we say that $f(g)$ satisfies $P$ for "almost all $g$ ", and abbreviate this by "a.a.g".

Definition 4. [23] Let $G$ be a discrete countable amenable semigroup with identity in which both right and left cancelation laws hold. $f \in w(G)$ is said to be statistically convergent to s, for any Folner sequence $\left\{S_{n}\right\}$ for $G$, if for every $\varepsilon>0$

$$
\lim _{n \rightarrow \infty} \frac{1}{\left|S_{n}\right|}\left|\left\{g \in S_{n}:|f(g)-s|\right\}\right|=0 .
$$

The set of all statistically convergent functions will be denoted by $S(G)$.

Definition 5. [28] Let $G$ be a discrete countable amenable semigroup with identity in which both right and left cancelation laws hold. $f \in w(G)$ is said to be $\mathscr{I}$-convergent to s for any Folner sequence $\left\{S_{n}\right\}$ for $G$, if for every $\varepsilon>0$;

$$
\left\{g \in S_{n}:|f(g)-s| \geq \varepsilon\right\} \in \mathscr{I} ;
$$

i.e., $|f(g)-s|<\varepsilon$ a.a.g. The set of all $\mathscr{I}$-convergent sequences will be denoted by $\mathscr{I}(G)$. 


\section{Main results}

Definition 6. Let $G$ be a discrete countable amenable semigroup with identity in which both right and left cancelation laws hold. The function $f \in w(G)$ is said to be $\mathscr{I}$-invariant convergent to s for any Folner sequence $\left\{S_{n}\right\}$ for $G$ if for every $\varepsilon>0$;

$$
\left\{g \in S_{n}:|f(g)-s| \geq \varepsilon\right\}
$$

belongs to $\mathscr{I}_{\sigma}$; i.e., $V\left(A_{\varepsilon}\right)=0$. The set of all $\mathscr{I}$-invariant convergent sequences will be denoted by $\mathscr{I}_{\sigma}(G)$.

Definition 7. Let $G$ be a discrete countable amenable semigroup with identity in which both right and left cancelation laws hold. The function $f \in w(G)$ is said to be invariant convergent to s for any Folner sequence $\left\{S_{n}\right\}$ for $G$ if

$$
\lim _{n \rightarrow \infty} \frac{1}{\left|S_{n}\right|} \sum_{1 \leq k \leq\left|S_{n}\right| \& g \in S_{n}} f\left(g_{\sigma^{k}(m)}\right)=s \text {, uniformly in } m \text {. }
$$

In this case, we write $f \rightarrow s\left(V_{\sigma}\right)$.

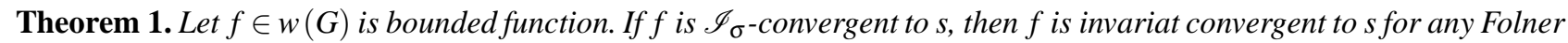
sequence $\left\{S_{n}\right\}$ for $G$.

Proof. Let $m, n \in \mathbb{N}$ be arbitrary, $\varepsilon>0$ and set

$$
L_{n}=\left\{g \in S_{n}:\left|f\left(g_{\sigma^{j}(m)}\right)-s\right| \geq \varepsilon\right\}, \text { uniformly in } m .
$$

For each $f \in w(G)$, we estimate

$$
t(m, n, f):=\left|\frac{f\left(g_{\sigma(m)}\right)+f\left(g_{\sigma^{2}(m)}\right)+\ldots+f\left(g_{\sigma^{n}(m)}\right)}{\left|S_{n}\right|}-s\right|
$$

We have

$$
t(m, n, f) \leq t^{(1)}(m, n, f)+t^{(2)}(m, n, f)
$$

where

$$
t^{(1)}(m, n, f):=\frac{1}{\left|S_{n}\right|} \sum_{1 \leq j \leq\left|S_{n}\right| \& g \in L_{n}}\left|f\left(g_{\sigma^{j}(m)}\right)-s\right|
$$

and

$$
t^{(2)}(m, n, x)=\frac{1}{\left|S_{n}\right|} \sum_{1 \leq j \leq\left|S_{n}\right| \& g \in S_{n} \backslash L_{n}}\left|f\left(g_{\sigma^{j}(m)}\right)-s\right| .
$$

Therefore, we have $t^{(2)}(m, n, f)<\varepsilon$ for each $f \in w(G)$ and for every $m=1,2, \ldots$. The boundedness of $f$ is implies that there exist $M>0$ such that

$$
\left|f\left(g_{\sigma^{j}(m)}\right)-s\right| \leq M,(j=1,2, \ldots ; m=1,2 \ldots),
$$

then this implies that

$$
\begin{aligned}
t^{(1)}(m, n, f) & \leq \frac{M}{\left|S_{n}\right|}\left|\left\{1<j<\left|S_{n}\right|:\left|f\left(g_{\sigma^{j}(m)}\right)-s\right| \geq \varepsilon\right\}\right| \\
& \leq M \cdot \frac{\max _{m}\left|\left\{1<j<\left|S_{n}\right|:: f_{k}\left(\left|f\left(g_{\sigma^{j}(m)}\right)-s\right|\right) \geq \varepsilon\right\}\right|}{\left|S_{n}\right|} \\
& =M \cdot \frac{K_{n}}{\left|S_{n}\right|} .
\end{aligned}
$$

Hence, $f$ is invariant convergent to $s$ for any Folner sequence $\left\{S_{n}\right\}$ for $G$. 
Definition 8. The function $f \in w(G)$ is said to be $\mathscr{I}^{*}$-invariat convergent to sfor any Folner sequence $\left\{S_{n}\right\}$ for $G$, if there exists a set

$$
M=\left\{m=\left(m_{i}\right): m_{i}<m_{i+1}, i \in \mathbb{N}\right\} \in \mathscr{F}\left(\mathscr{I}_{\sigma}\right)
$$

such that

$$
\lim _{k \rightarrow \infty} f\left(g_{m_{k}}\right)=s .
$$

The set of all $\mathscr{I}^{*}$-invariat convergent sequences will be denoted by $\mathscr{I}_{\sigma}^{*}(G)$.

Theorem 2. If the function $f \in w(G)$ is $\mathscr{I}^{*}$-invariat convergent to s, the function is $\mathscr{I}$-invariant convergent to s for any Folner sequence $\left\{S_{n}\right\}$ for $G$.

Proof. By assumption, there exists a set $H \in \mathscr{I}_{\sigma}$ such that for $M=N \backslash H=M=\left\{m=\left(m_{i}\right): m_{i}<m_{i+1}, i \in \mathbb{N}\right\}$ we have

$$
\lim _{k \rightarrow \infty} f\left(g_{m_{k}}\right)=s,
$$

Let $\varepsilon>0$ by (1), there exists $k_{0} \in \mathbb{N}$ such that

$$
\left|f\left(g_{m_{k}}\right)-s\right|<\varepsilon,
$$

for each $k>k_{0}$. Then, obviously

$$
\left\{k \in \mathbb{N}:\left|f\left(g_{m_{k}}\right)-s\right| \geq \varepsilon\right\} \subset H \cup\left\{m_{1}<m_{2}<\ldots<m_{k_{0}}\right\} .
$$

Since $\mathscr{I}_{\sigma}$ is admissible, the set on the right-hand side of (2) belongs to $\mathscr{I}_{\sigma}$. So $f$ is $\mathscr{I}$-invariant convergent to $s$ for any Folner sequence $\left\{S_{n}\right\}$ for $G$.

Definition 9. The function $f \in w(G)$ is said to be p-strongly invariant convergent to s for any Folner sequence $\left\{S_{n}\right\}$ for $G$ if

$$
\lim _{n \rightarrow \infty} \frac{1}{\left|S_{n}\right|} \sum_{1 \leq k \leq\left|S_{n}\right| \& g \in S_{n}}\left|f\left(g_{\sigma^{k}(m)}\right)-s\right|^{p}=0 \text {, uniformly in } m,
$$

where $0<p<\infty$. In this case, we write $f \rightarrow s\left[V_{\sigma}\right]_{p}$.

Theorem 3. Let $\mathscr{I}_{\sigma} \subset 2^{\mathbb{N}}$ be an admissible ideal and $0<p<\infty$.

(i) If $f \rightarrow s\left[V_{\sigma}\right]_{p}$, then $f \rightarrow s\left(\mathscr{I}_{\sigma}\right)$.

(ii) If $f \in w(G)$ is bounded and $f \rightarrow s\left(\mathscr{I}_{\sigma}\right)$, then $f \rightarrow s\left[V_{\sigma}\right]_{p}$.

(iii) If $f \in w(G)$, then $f$ is $\mathscr{I}_{\sigma}$-convergent if and only if $f \rightarrow s\left[V_{\sigma}\right]_{p}$.

Proof. (i) Let $f \rightarrow s\left[V_{\sigma}\right]_{p}, 0<p<\infty$. Suppose $\varepsilon>0$. Then for every $m \in \mathbb{N}$, we have

$$
\begin{aligned}
\sum_{1<j<\left|S_{n}\right| \& g \in S_{n}}\left|f\left(g_{\sigma^{j}(m)}\right)-s\right|^{p} & \geq \sum_{1<j<\left|S_{n}\right| \&\left|f\left(g_{\sigma^{j}(m)}\right)-s\right| \geq \varepsilon}\left|f\left(g_{\sigma^{j}(m)}\right)-s\right|^{p} \\
& \geq \varepsilon^{p} \cdot\left|\left\{1<j<\left|S_{n}\right|:\left|f\left(g_{\sigma^{j}(m)}\right)-s\right| \geq \varepsilon\right\}\right| \\
& \geq \varepsilon^{p} \cdot \max _{m}\left|\left\{1<j<\left|S_{n}\right|:\left|f\left(g_{\sigma^{j}(m)}\right)-s\right| \geq \varepsilon\right\}\right|
\end{aligned}
$$

and

$$
\begin{aligned}
\frac{1}{\left|S_{n}\right|} \sum_{1<j<\left|S_{n}\right| \& g \in S_{n}}\left|f\left(g_{\sigma^{j}(m)}\right)-s\right|^{p} & \geq \varepsilon^{p} \cdot \frac{\max _{m}\left|\left\{1<j<\left|S_{n}\right| \& g \in S_{n}:\left|f\left(g_{\sigma^{k}(m)}\right)-s\right| \geq \varepsilon\right\}\right|}{\left|S_{n}\right|} \\
& =\varepsilon^{p} \cdot \frac{K_{n}}{\left|S_{n}\right|}
\end{aligned}
$$


for every $m=1,2, \ldots$ This implies $\lim _{n \rightarrow \infty} \frac{K_{n}}{\left|S_{n}\right|}=0$ and so $f \rightarrow s\left(\mathscr{I}_{\sigma}\right)$.

(ii) Now suppose that $f \in w(G)$ is bounded and $f \rightarrow s\left(\mathscr{I}_{\sigma}\right)$. Let $0<p<\infty$ and $\varepsilon>0$. By assumption, we have $V\left(A_{\varepsilon}\right)=0$. The boundedness of $f \in w(G)$ implies that there exist $M>0$ such that

$$
\left|f\left(g_{\sigma^{j}(m)}\right)-s\right| \leq M, j=1,2, \ldots ; m=1,2, \ldots
$$

Observe that for every $n \in \mathbb{N}$ we have that

$$
\begin{aligned}
\frac{1}{\left|S_{n}\right|} \sum_{1<j<\left|S_{n}\right| \& g \in S_{n}}\left|f\left(g_{\sigma^{j}(m)}\right)-s\right|^{p} & =\frac{1}{\left|S_{n}\right|} \sum_{1<j<\left|S_{n}\right| \&\left|f\left(g_{\sigma^{j}(m)}\right)-s\right| \geq \varepsilon}\left|f\left(g_{\sigma^{j}(m)}\right)-s\right|^{p}+\sum_{1<j<\left|S_{n}\right| \&\left|f\left(g_{\sigma^{j}(m)}\right)-s\right|<\varepsilon}\left|f\left(g_{\sigma^{j}(m)}\right)-s\right|^{p} \\
& \leq M \cdot \frac{\max _{m}\left|\left\{1 \leq j \leq\left|S_{n}\right|:\left|f\left(g_{\sigma^{j}(m)}\right)-s\right| \geq \varepsilon\right\}\right|}{\left|S_{n}\right|}+\varepsilon^{p} \\
& \leq M \cdot \frac{K_{n}}{\left|S_{n}\right|}+\varepsilon^{p}
\end{aligned}
$$

for each $f \in w(G)$.

Hence, we obtain

$$
\lim _{n \rightarrow \infty} \frac{1}{\left|S_{n}\right|} \sum_{1<j<\left|S_{n}\right| \& g \in S_{n}}\left|f\left(g_{\sigma^{j}(m)}\right)-s\right|^{p}=0, \text { uniformly in } m .
$$

(iii) This is immediate consequence of (i) and (ii).

Definition 10. The function $f \in w(G)$ is said to be $\mathscr{I}$-lacunary invariant statistically convergent to $s$ for any Folner sequence $\left\{S_{n}\right\}$ for $G$ for each $\varepsilon>0$ and $\delta>0$,

$$
\left\{r \in \mathbb{N}: \frac{1}{h_{r}}\left|\left\{k \in I_{r} \& g \in S_{n}:\left|f\left(g_{\sigma^{k}(m)}\right)-s\right| \geq \varepsilon\right\}\right| \geq \delta\right\} \in \mathscr{I}_{\sigma} \text {, uniformly in } m \text {. }
$$

In this case we write $f \rightarrow s\left(S_{\sigma \theta}(\mathscr{I})\right)$.

Definition 11. The function $f \in w(G)$ is said to be strongly $\mathscr{I}$-lacunary invariant convergent to s for any Folner sequence $\left\{S_{n}\right\}$ for $G$ for each $\varepsilon>0$,

$$
\left\{r \in \mathbb{N}: \frac{1}{h_{r}} \sum_{k \in I_{r} \& g \in S_{n}}\left|f\left(g_{\sigma^{k}(m)}\right)-s\right| \geq \varepsilon\right\} \in \mathscr{I}_{\sigma} \text {, uniformly in } m .
$$

In this case we write $f \rightarrow s\left(N_{\sigma \theta}(\mathscr{I})\right)$.

We shall denote by $S_{\sigma \theta}(\mathscr{I}), N_{\sigma \theta}(\mathscr{I})$ the collections of all $\mathscr{I}$-lacunary invariant statistically convergent and strongly $\mathscr{I}$-lacunary invariant functions for the function $f \in w(G)$, respectively.

Theorem 4. Let $\theta=\left\{k_{r}\right\}$ be a lacunary sequence and $f \in w(G)$ be a function in $S$.

(i) If $f \rightarrow s\left(N_{\sigma \theta}(\mathscr{I})\right)$ then $f \rightarrow s\left(S_{\sigma \theta}(\mathscr{I})\right)$.

(ii) If $f \in w(G)$ is bounded function and $f \rightarrow s\left(S_{\sigma \theta}(\mathscr{I})\right)$ then $f \rightarrow s\left(N_{\sigma \theta}(\mathscr{I})\right)$.

Proof. (i) Let $\varepsilon>0$ and $f \rightarrow s\left(N_{\sigma \theta}(\mathscr{I})\right)$. Then we can write

$$
\sum_{k \in I_{r} \& g \in S_{n}}\left|f\left(g_{\sigma^{k}(m)}\right)-s\right| \geq \sum_{k \in I_{r}, g \in S_{n} \&\left|f\left(g_{\sigma^{k}(m)}\right)-s\right| \geq \varepsilon}\left|f\left(g_{\sigma^{k}(m)}\right)-s\right| \geq \varepsilon .\left|\left\{k \in I_{r} \& g \in S_{n}:\left|f\left(g_{\sigma^{k}(m)}\right)-s\right| \geq \varepsilon\right\}\right| .
$$


So for given $\delta>0$,

$$
\frac{1}{h_{r}}\left|\left\{k \in I_{r} \& g \in S_{n}:\left|f\left(g_{\sigma^{k}(m)}\right)-s\right| \geq \varepsilon\right\}\right| \geq \delta \Longrightarrow \frac{1}{h_{r}} \sum_{k \in I_{r}, g \in S_{n}}\left|f\left(g_{\sigma^{k}(m)}\right)-s\right| \geq \varepsilon . \delta,
$$

i.e.

$$
\left\{r \in \mathbb{N}: \frac{1}{h_{r}}\left|\left\{k \in I_{r} \& g \in S_{n}:\left|f\left(g_{\sigma^{k}(m)}\right)-s\right| \geq \varepsilon\right\}\right| \geq \delta\right\} \subseteq\left\{r \in \mathbb{N}: \frac{1}{h_{r}} \sum_{k \in I_{r}, g \in S_{n}}\left|f\left(g_{\sigma^{k}(m)}\right)-s\right| \geq \varepsilon . \delta\right\} .
$$

Since $f \rightarrow s\left(N_{\sigma \theta}(\mathscr{I})\right)$, the set on the right-hand side belongs to $\mathscr{I}_{\sigma}$ and so it follows that $f \rightarrow s\left(S_{\sigma \theta}(\mathscr{I})\right)$.

(ii) Suppose that $f \in w(G)$ is bounded function and $f \rightarrow s\left(S_{\sigma \theta}(\mathscr{I})\right)$. Then we can assume that

$$
\left|f\left(g_{\sigma^{k}(m)}\right)-s\right| \leq M
$$

for each $k \in I_{r}$ and $g \in S_{n}$.

Given $\varepsilon>0$, we get

$$
\begin{aligned}
\frac{1}{h_{r}} \sum_{k \in I_{r}, g \in S_{n}}\left|f\left(g_{\sigma^{k}(m)}\right)-s\right| & =\frac{1}{h_{r}} \sum_{\substack{k \in I_{r}, g \in S_{n} \\
\left|f\left(g_{\sigma^{k}(m)}\right)-s\right| \geq \varepsilon}} f_{k}\left(\left|A_{k}\left(x_{\sigma^{k}(m)}\right)-L\right|\right)+\frac{1}{h_{r}} \sum_{\substack{k \in I_{r}, g \in S_{n} \\
\left|f\left(g_{\sigma^{k}(m)}\right)-s\right|<\varepsilon}} f_{k}\left(\left|A_{k}\left(x_{\sigma^{k}(m)}\right)-L\right|\right) \\
& \leq \frac{M}{h_{r}}\left|\left\{k \in I_{r}, g \in S_{n}:\left|f\left(g_{\sigma^{k}(m)}\right)-s\right| \geq \varepsilon\right\}\right|+\varepsilon .
\end{aligned}
$$

Note that

$$
A(\varepsilon)=\left\{r \in \mathbb{N}: \frac{1}{h_{r}}\left|\left\{k \in I_{r}, g \in S_{n}:\left|f\left(g_{\sigma^{k}(m)}\right)-s\right| \geq \varepsilon\right\}\right| \geq \frac{\varepsilon}{M}\right\} \in \mathscr{I}_{\sigma} .
$$

If $r \in(A(\varepsilon))^{c}$ then

$$
\frac{1}{h_{r}} \sum_{k \in I_{r}, g \in S_{n}}\left|f\left(g_{\sigma^{k}(m)}\right)-s\right|<2 \varepsilon
$$

Hence

$$
\left\{r \in \mathbb{N}: \frac{1}{h_{r}} \sum_{k \in I_{r}, g \in S_{n}}\left|f\left(g_{\sigma^{k}(m)}\right)-s\right| \geq 2 \varepsilon\right\} \subset A(\varepsilon)
$$

and so belongs to $\mathscr{I}_{\sigma}$. This shows that $f \rightarrow s\left(N_{\sigma \theta}(\mathscr{I})\right)$. This completes the proof.

Definition 12. The function $f \in w(G)$ is said to be $\mathscr{I}_{\sigma}$-statistically convergent to s for any Folner sequence $\left\{S_{n}\right\}$ for $G$ if for each $\varepsilon>0$ and $\delta>0$,

$$
\left\{g \in S_{n}: \frac{1}{\left|S_{n}\right|}\left|\left\{k \leq\left|S_{n}\right|:\left|f\left(g_{\sigma^{k}(m)}\right)-s\right| \geq \varepsilon\right\}\right| \geq \delta\right\} \in \mathscr{I}_{\sigma}
$$

In this case we write $f \rightarrow s\left(S\left(\mathscr{I}_{\sigma}\right)\right)$.

Theorem 5. If $\theta=\left\{k_{r}\right\}$ be a lacunary sequence with $\liminf _{r} q_{r}>1$, then

$$
f \rightarrow s\left(S\left(\mathscr{I}_{\sigma}\right)\right) \Rightarrow f \rightarrow s\left(S_{\sigma \theta}(\mathscr{I})\right) .
$$


Proof. Suppose first that $\liminf _{r} q_{r}>1$, then there exists a $\alpha>0$ such that $q_{r} \geq 1+\alpha$ for sufficiently large $r$, which implies that

$$
\frac{h_{r}}{k_{r}} \geq \frac{\alpha}{1+\alpha}
$$

If $f \rightarrow s\left(S\left(\mathscr{I}_{\sigma}\right)\right)$, then for every $\varepsilon>0$, for each $x \in X$ and for sufficiently large $r$, we have

$$
\begin{aligned}
\frac{1}{k_{r}}\left|\left\{g \in S_{k_{r}}:\left|f\left(g_{\sigma^{k}(m)}\right)-s\right| \geq \varepsilon\right\}\right| & \geq \frac{1}{k_{r}}\left|\left\{k \in I_{r}, g \in S_{n}:\left|f\left(g_{\sigma^{k}(m)}\right)-s\right| \geq \varepsilon\right\}\right| \\
& \geq \frac{\alpha}{1+\alpha} \frac{1}{h_{r}}\left|\left\{k \in I_{r}, g \in S_{n}:\left|f\left(g_{\sigma^{k}(m)}\right)-s\right| \geq \varepsilon\right\}\right| ;
\end{aligned}
$$

Then for any $\delta>0$, we get

$$
\left\{r \in \mathbb{N}: \frac{1}{h_{r}}\left|\left\{k \in I_{r}:\left|f\left(g_{\sigma^{k}(m)}\right)-s\right| \geq \varepsilon\right\}\right| \geq \delta\right\} \subseteq\left\{r \in \mathbb{N}: \frac{1}{k_{r}}\left|\left\{g \in S_{k_{r}}:\left|f\left(g_{\sigma^{k}(m)}\right)-s\right| \geq \varepsilon\right\}\right| \geq \frac{\delta \alpha}{(\alpha+1)}\right\} \in \mathscr{I}_{\sigma}
$$

This completes the proof.

Theorem 6. If $\theta=\left\{k_{r}\right\}$ be a lacunary sequence with $\limsup _{r} q_{r}<\infty$, then

$$
f \rightarrow s\left(S_{\sigma \theta}(\mathscr{I})\right) \Rightarrow f \rightarrow s\left(S\left(\mathscr{I}_{\sigma}\right)\right)
$$

Proof. If limsup $q_{r}<\infty$ then without any loss of generality we can assume that there exists a $K>0$ such that $q_{r}<K$ for all $r \geq 1$. Let $f \rightarrow s\left(S_{\sigma \theta}(\mathscr{I})\right)$ and for $\delta>0$. Then there exists $B>0$ and $\varepsilon>0$ such that for every $j \geq B$

$$
M_{j}=\frac{1}{h_{j}}\left|\left\{k \in I_{j}, g \in S_{n}:\left|f\left(g_{\sigma^{k}(m)}\right)-s\right| \geq \varepsilon\right\}\right|<\delta
$$

Also we can find $A>0$ such that $M_{j}<A$ for all $j=1,2, \ldots$. Now, let $n \in \mathbb{N}$ be an integer satisfying $k_{r-1}<\left|S_{n}\right| \leq k_{r}$ where $r>B$. Then, we can write

$$
\begin{aligned}
& \frac{1}{\left|S_{n}\right|}\left|\left\{k \leq\left|S_{n}\right|:\left|f\left(g_{\sigma^{k}(m)}\right)-s\right| \geq \varepsilon\right\}\right| \leq \frac{1}{k_{r-1}}\left|\left\{k \leq\left|S_{k_{r}}\right|:\left|f\left(g_{\sigma^{k}(m)}\right)-s\right| \geq \varepsilon\right\}\right| \\
& =\frac{1}{k_{r-1}}\left|\left\{k \in I_{1}:\left|f\left(g_{\sigma^{k}(m)}\right)-s\right| \geq \varepsilon\right\}\right|+\frac{1}{k_{r-1}}\left|\left\{k \in I_{2}:\left|f\left(g_{\sigma^{k}(m)}\right)-s\right| \geq \varepsilon\right\}\right|+\ldots \\
& +\frac{1}{k_{r-1}}\left|\left\{k \in I_{r}:\left|f\left(g_{\sigma^{k}(m)}\right)-s\right| \geq \varepsilon\right\}\right|=\frac{k_{1}}{k_{r-1} k_{1}}\left|\left\{k \in I_{1}:\left|f\left(g_{\sigma^{k}(m)}\right)-s\right| \geq \varepsilon\right\}\right| \\
& +\frac{k_{2}-k_{1}}{k_{r-1}\left(k_{2}-k_{1}\right)}\left|\left\{k \in I_{2}:\left|f\left(g_{\sigma^{k}(m)}\right)-s\right| \geq \varepsilon\right\}\right|+\ldots+\frac{k_{B}-k_{B-1}}{k_{r-1}\left(k_{B}-k_{B-1}\right)}\left|\left\{k \in I_{B}:\left|f\left(g_{\sigma^{k}(m)}\right)-s\right| \geq \varepsilon\right\}\right| \\
& +\ldots+\frac{k_{r}-k_{r-1}}{k_{r-1}\left(k_{r}-k_{r-1}\right)}\left|\left\{k \in I_{r}:\left|f\left(g_{\sigma^{k}(m)}\right)-s\right| \geq \varepsilon\right\}\right|=\frac{k_{1}}{k_{r-1}} M_{1}+\frac{k_{2}-k_{1}}{k_{r-1}} M_{2}+\ldots+\frac{k_{B}-k_{B-1}}{k_{r-1}} M_{B} \\
& +\ldots+\frac{k_{r}-k_{r-1}}{k_{r-1}} M_{r} \leq\left\{\sup _{i \geq 1} M_{i}\right\} \frac{k_{B}}{k_{r-1}}+\left\{\sup _{i \geq B} M_{i}\right\} \frac{k_{r}-k_{B}}{k_{r-1}} \leq A_{\frac{k_{B}}{k_{r-1}}}+\delta K .
\end{aligned}
$$

This completes the proof of the theorem.

Combining Theorem 5 and Theorem 6 we have 
Theorem 7. If $\theta=\left\{k_{r}\right\}$ be a lacunary sequence with $1<\liminf _{r} q_{r} \leq \limsup _{r} q_{r}<\infty$, then

$$
f \rightarrow s\left(S_{\sigma \theta}(\mathscr{I})\right) \Leftrightarrow f \rightarrow s\left(S\left(\mathscr{I}_{\sigma}\right)\right)
$$

Proof. This is an immediate consequence of Theorem 5 and Theorem 6.

Definition 13. The function $f \in w(G)$ is said to be strongly Cesàro $\mathscr{I}_{\sigma}$-summable to s for any Folner sequence $\left\{S_{n}\right\}$ for $G$ iffor each $\varepsilon>0$,

$$
\left\{g \in S_{n}: \frac{1}{\left|S_{n}\right|} \sum_{1 \leq k \leq\left|S_{n}\right| \& g \in S_{n}}\left|f\left(g_{\sigma^{k}(m)}\right)-s\right| \geq \varepsilon\right\} \in \mathscr{I}_{\sigma}
$$

uniformly in $m$. (denoted by $\left.f \rightarrow s\left[C_{1}\left(\mathscr{I}_{\sigma}\right)\right]\right)$.

Definition 14. The function $f \in w(G)$ is said to be strongly $\lambda_{\mathscr{I}}$-invariant convergent to sor any Folner sequence $\left\{S_{n}\right\}$ for $G$ if for each $\varepsilon>0$,

$$
\left\{g \in S_{n}: \frac{1}{\lambda_{n}} \sum_{k \in I_{n}, g \in S_{n}}\left|f\left(g_{\sigma^{k}(m)}\right)-s\right| \geq \varepsilon\right\} \in \mathscr{I}_{\sigma}
$$

uniformly in $m$, where $I_{n}=\left[n-\lambda_{n}+1, n\right] .\left(\right.$ denoted by $\left.f \rightarrow s\left(V_{\lambda}\left(\mathscr{I}_{\sigma}\right)\right)\right)$.

Definition 15. The function $f \in w(G)$ is said to be $\mathscr{I}_{\sigma}$ - $\lambda$-statistically convergent to sfor any Folner sequence $\left\{S_{n}\right\}$ for $G$ iffor each $\varepsilon>0$, for each $\delta>0$,

$$
\left\{g \in S_{n}: \frac{1}{\lambda_{n}}\left|\left\{k \in I_{n}:\left|f\left(g_{\sigma^{k}(m)}\right)-s\right| \geq \varepsilon\right\}\right| \geq \delta\right\} \in \mathscr{I}_{\sigma}
$$

uniformly in $m$. (denoted by $\left.f \rightarrow s\left(S_{\lambda}\left(\mathscr{I}_{\sigma}\right)\right)\right)$.

Theorem 8. Let $\lambda \in \Lambda$ and $\mathscr{I}_{\sigma}$ is an admissible ideal in $\mathbb{N}$. If $f \rightarrow s\left(V_{\lambda}\left(\mathscr{I}_{\sigma}\right)\right)$, then $f \rightarrow s\left(S_{\lambda}\left(\mathscr{I}_{\sigma}\right)\right)$.

Proof. Assume that $f \rightarrow s\left(V_{\lambda}\left(\mathscr{I}_{\sigma}\right)\right)$ and $\varepsilon>0$. Then,

$$
\sum_{k \in I_{n}, g \in S_{n}}\left|f\left(g_{\sigma^{k}(m)}\right)-s\right| \geq \sum_{\substack{k \in I_{n}, g \in S_{n} \\ f\left(g\left(g_{\sigma^{k}(m)}\right)-s \mid \geq \varepsilon\right.}}\left|f\left(g_{\sigma^{k}(m)}\right)-s\right| \geq \varepsilon .\left|\left\{k \in I_{n}, g \in S_{n}:\left|f\left(g_{\sigma^{k}(m)}\right)-s\right| \geq \varepsilon\right\}\right|
$$

and so,

$$
\frac{1}{\varepsilon \lambda_{n}} \sum_{k \in I_{n}, g \in S_{n}}\left|f\left(g_{\sigma^{k}(m)}\right)-s\right| \geq \frac{1}{\lambda_{n}}\left|\left\{k \in I_{n}, g \in S_{n}:\left|f\left(g_{\sigma^{k}(m)}\right)-s\right| \geq \varepsilon\right\}\right| .
$$

Then for any $\delta>0$,

$$
\begin{aligned}
\left\{g \in S_{n}: \frac{1}{\lambda_{n}} \mid\left\{k \in I_{n}:\right.\right. & \left.\left.\left|f\left(g_{\sigma^{k}(m)}\right)-s\right| \geq \varepsilon\right\} \mid \geq \delta\right\} \\
& \subseteq\left\{g \in S_{n}: \frac{1}{\lambda_{n}} \sum_{k \in I_{n}, g \in S_{n}}\left|f\left(g_{\sigma^{k}(m)}\right)-s\right| \geq \varepsilon \delta\right\} .
\end{aligned}
$$

Since right hand belongs to $\mathscr{I}_{\sigma}$ then left hand also belongs to $\mathscr{I}_{\sigma}$ and this completes the proof.

Theorem 9. If $\liminf \frac{\lambda_{n}}{\left|S_{n}\right|}>0$ then $f \rightarrow s\left(S\left(\mathscr{I}_{\sigma}\right)\right)$ implies $f \rightarrow s\left(S_{\lambda}\left(\mathscr{I}_{\sigma}\right)\right)$. 
Proof. Assume that $\liminf \frac{\lambda_{n}}{\left|S_{n}\right|}>0$ there exists a $\delta>0$ such that $\frac{\lambda_{n}}{\left|S_{n}\right|} \geq \delta$ for sufficiently large $n$. For given $\varepsilon>0$ we have,

$$
\frac{1}{\left|S_{n}\right|}\left\{k \leq\left|S_{n}\right|:\left|f\left(g_{\sigma^{k}(m)}\right)-s\right| \geq \varepsilon\right\} \supseteq \frac{1}{\left|S_{n}\right|}\left\{k \in I_{n}:\left|f\left(g_{\sigma^{k}(m)}\right)-s\right| \geq \varepsilon\right\} \text {. }
$$

Therefore,

$$
\begin{aligned}
\frac{1}{\left|S_{n}\right|}\left|\left\{k \leq\left|S_{n}\right|:\left|f\left(g_{\sigma^{k}(m)}\right)-s\right| \geq \varepsilon\right\}\right| & \geq \frac{1}{\left|S_{n}\right|}\left|\left\{k \in I_{n}:\left|f\left(g_{\sigma^{k}(m)}\right)-s\right| \geq \varepsilon\right\}\right| \\
& \geq \frac{\lambda_{n}}{\left|S_{n}\right|} \frac{1}{\lambda_{n}}\left|\left\{k \in I_{n}:\left|f\left(g_{\sigma^{k}(m)}\right)-s\right| \geq \varepsilon\right\}\right| \\
& \geq \delta \cdot \frac{1}{\lambda_{n}}\left|\left\{k \in I_{n}:\left|f\left(g_{\sigma^{k}(m)}\right)-s\right| \geq \varepsilon\right\}\right|
\end{aligned}
$$

then for any $\eta>0$ we get

$$
\begin{aligned}
\left\{g \in S_{n}:\right. & \left.\frac{1}{\lambda_{n}}\left|\left\{k \in I_{n}:\left|f\left(g_{\sigma^{k}(m)}\right)-s\right| \geq \varepsilon\right\}\right| \geq \eta\right\} \\
& \subseteq\left\{g \in S_{n}: \frac{1}{\left|S_{n}\right|}\left|\left\{k \leq\left|S_{n}\right|:\left|f\left(g_{\sigma^{k}(m)}\right)-s\right| \geq \varepsilon\right\}\right| \geq \eta \delta\right\} \in \mathscr{I}_{\sigma}
\end{aligned}
$$

and this completes the proof.

Theorem 10. If $\lambda=\left(\lambda_{n}\right) \in \Delta$ be such that $\lim _{n \rightarrow \infty} \frac{\lambda_{n}}{\left|S_{n}\right|}=1$, then $S_{\lambda}\left(\mathscr{I}_{\sigma}\right) \subset S\left(\mathscr{I}_{\sigma}\right)$.

Proof. Let $\delta>0$ be given. Since $\lim _{n \rightarrow \infty} \frac{\lambda_{n}}{\left|S_{n}\right|}=1$, we can choose $m \in N$ such that $\left|\frac{\lambda_{n}}{\left|S_{n}\right|}-1\right|<\frac{\delta}{2}$, for all $n \geq m$. Now observe that, for $\varepsilon>0$

$$
\begin{aligned}
\frac{1}{\left|S_{n}\right|}\left|\left\{k \leq\left|S_{n}\right|:\left|f\left(g_{\sigma^{k}(m)}\right)-s\right| \geq \varepsilon\right\}\right| & =\frac{1}{\left|S_{n}\right|}\left|\left\{k \leq\left|S_{n}\right|-\lambda_{n}:\left|f\left(g_{\sigma^{k}(m)}\right)-s\right| \geq \varepsilon\right\}\right| \\
& +\frac{1}{\left|S_{n}\right|}\left|\left\{k \in I_{n}:\left|f\left(g_{\sigma^{k}(m)}\right)-s\right| \geq \varepsilon\right\}\right| \\
& \leq \frac{\left|S_{n}\right|-\lambda_{n}}{\left|S_{n}\right|}+\frac{1}{\left|S_{n}\right|}\left|\left\{k \in I_{n}:\left|f\left(g_{\sigma^{k}(m)}\right)-s\right| \geq \varepsilon\right\}\right| \\
& \leq 1-\left(1-\frac{\delta}{2}\right)+\frac{1}{\left|S_{n}\right|}\left|\left\{k \in I_{n}:\left|f\left(g_{\sigma^{k}(m)}\right)-s\right| \geq \varepsilon\right\}\right| \\
& =\frac{\delta}{2}+\frac{1}{\left|S_{n}\right|}\left|\left\{k \in I_{n}:\left|f\left(g_{\sigma^{k}(m)}\right)-s\right| \geq \varepsilon\right\}\right|,
\end{aligned}
$$

for all $n \geq m$. Hence

$$
\begin{aligned}
& \left\{g \in S_{n}: \frac{1}{\left|S_{n}\right|}\left|\left\{k \leq\left|S_{n}\right|:\left|f\left(g_{\sigma^{k}(m)}\right)-s\right| \geq \varepsilon\right\}\right| \geq \delta\right\} \\
& \quad \subset\left\{g \in S_{n}: \frac{1}{\left|S_{n}\right|}\left|\left\{k \in I_{n}:\left|f\left(g_{\sigma^{k}(m)}\right)-s\right| \geq \varepsilon\right\}\right| \geq \frac{\delta}{2}\right\} \cup\{1,2, \ldots, m\}
\end{aligned}
$$

If $f$ is $\mathscr{I}_{\sigma}$ - $\lambda$-statistically convergent to $s$, then the set on the right hand side belongs to $\mathscr{I}_{\sigma}$ and so the set on the left hand side also belongs to $\mathscr{I}_{\sigma}$. This shows that $f$ is $\mathscr{I}_{\sigma}$-statistically convergent to $s$. 
Theorem 11. If $f \rightarrow s\left(V_{\lambda}\left(\mathscr{I}_{\sigma}\right)\right)$ is then $f \rightarrow s\left[C_{1}\left(\mathscr{I}_{\sigma}\right)\right]$.

Proof. Assume that $f \rightarrow s\left(V_{\lambda}\left(\mathscr{I}_{\sigma}\right)\right)$ and $\varepsilon>0$. Then,

$$
\begin{aligned}
\frac{1}{\left|S_{n}\right|} \sum_{g \in S_{n}}\left|f\left(g_{\sigma^{k}(m)}\right)-s\right| & =\frac{1}{\left|S_{n}\right|} \sum_{k=1}^{\left|S_{n}\right|-\lambda_{n}}\left|f\left(g_{\sigma^{k}(m)}\right)-s\right|+\frac{1}{\left|S_{n}\right|} \sum_{k \in I_{n}, g \in S_{n}}\left|f\left(g_{\sigma^{k}(m)}\right)-s\right| \\
& \leq \frac{1}{\lambda_{n}} \sum_{k=1}^{\left|S_{n}\right|-\lambda_{n}}\left|f\left(g_{\sigma^{k}(m)}\right)-s\right|+\frac{1}{\lambda_{n}} \sum_{k \in I_{n}, g \in S_{n}}\left|f\left(g_{\sigma^{k}(m)}\right)-s\right| \\
& \leq \frac{2}{\lambda_{n}} \sum_{k \in I_{n}, g \in S_{n}}\left|f\left(g_{\sigma^{k}(m)}\right)-s\right|
\end{aligned}
$$

and so,

$$
\left\{g \in S_{n}: \frac{1}{\left|S_{n}\right|} \sum_{g \in S_{n}}\left|f\left(g_{\sigma^{k}(m)}\right)-s\right| \geq \varepsilon\right\} \subseteq\left\{g \in S_{n}: \frac{1}{\lambda_{n}} \sum_{k \in I_{n}, g \in S_{n}}\left|f\left(g_{\sigma^{k}(m)}\right)-s\right| \geq \frac{\varepsilon}{2}\right\}
$$

belongs to $\mathscr{I}_{\sigma}$. Hence $f \rightarrow s\left[C_{1}\left(\mathscr{I}_{\sigma}\right)\right]$.

\section{Competing interests}

The authors declare that they have no competing interests.

\section{Authors' contributions}

All authors have contributed to all parts of the article. All authors read and approved the final manuscript.

\section{References}

[1] M. Day, Amenable semigroups, Illinois J. Math. 1 (1957) 509-544.

[2] P. Das, E. Savaş and S. Kr. Ghosal, On generalized of certain summability methods using ideals, Appl. Math. Letter, 36 (2011) 1509-1514.

[3] S.A. Douglass, On a concept of summability in amenable semigroups, Math. Scand. 28 (1968) 96-102.

[4] S.A. Douglass, Summing sequences for amenable semigroups, Michigan Math J. 20 (1973) 169-179.

[5] H. Fast, Sur la convergence statistique, Colloq. Math. 2 (1951) 241-244.

[6] J. A Fridy, Orhan C., (1993). Lacunary Statistical Convergence. Pacific Journal of Mathematics, 160(1), 43-51.

[7] A. R. Freedman and J. J. Sember, Densities and summability, Pacitific J. Math., 95(1981), 10-11.

[8] Freedman, A. R., Sember, J. J., \& Raphael, Marc. (1978). Some Cesaro type summability spaces. Proceedings of The London Mathematical Society, 37, 508-520.

[9] Lorentz G. G., A contribution to the theory of divergent sequences, Acta Math. 80 (1948), 167-190.

[10] P. Kostyrko, T. Salát, W. Wilezyński, $\mathscr{I}$-Convergence, Real Analysis Exchange Vol. 26(2), pp. 669-686, 2000.

[11] T. Salát, On statistically convergent sequences of real numbers, Math. Slovaca 30 (1980), 139-150.

[12] T. Salát, B.C. Tripathy, and M. Ziman, On Some Properties of $\mathscr{I}$-Convergence, Tatra Mt. Math. Publ.28, pp. 279-286 (2004).

[13] T. Salát, B.C. Tripathy, and M. Ziman, $\mathscr{I}$-Convergence Field, Tatra Mt. Math. Publ.28, pp. 279-286 (2005).

[14] L. Leindler. "Uber die de la Vall'ee-Pousnsche Summierbarkeit allge meiner orthogonalreihen." Acta Math. Acad. Sci. Hungarica, vol. 16, pp. 375-387, 1965.

[15] P.F. Mah, Summability in amenable semigroups, Trans. Amer. Math. Soc. 156 (1971) 391-403. 
[16] P.F. Mah, Matrix summability in amenable semigroups, Proc. Amer. Math. Soc. 36 (1972) 414-420.

[17] M. Mursaleen, $\lambda$-Statistical Convergence, Math. Slovaca, 50 No. 1 (2000) pp. 111-115.

[18] M. Mursaleen, Invariant means and some matrix transformations, Tamkang J. Math. 10 (1979), 183-188.

[19] Savaş E., On lacunary strong $\sigma$-convergence, Indian J. Pure Appl. Math. 21 (1990), 359-365.

[20] Savaş E., Strongly $\sigma$-convergent sequences, Bull. Calcutta Math. 81(1989), 295-300.

[21] Savaş E., Das P., A generalized statistical convergence via ideals, Applied Mathematics Letters 24 (2011) 826-830.

[22] Savaş E., Nuray F., On sigma-statistically convergence and lacunary sigma-statistically convergence, Mathematica Slovaca, Vol. 43 (1993), No. 3, 309-315.

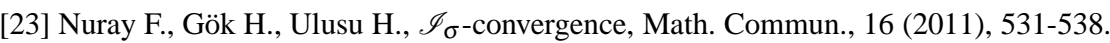

[24] Nuray F., Savaş E., Invariant statistical convergence and $A$-invariant statistical convergence, Indian J. Pure and Appl. Math. 10(1994), 267-274.

[25] Nuray F., Rhoades B.E., Some kinds of convergence defined by Folner sequences, Analysis 31 (4) 381-390 (2011).

[26] I. Nomika, Folner's conditions for amenable semigroups, Math. Scand. 15 (1964) 18-28.

[27] Schoenberg, I. J. (1959). The integrability of certain functions and related summability methods, Amer. Math. Monthly, 66, 361375.

[28] Kişi Ö. A generalized statistical convergence via ideal defined by folner sequence on amenable semigroups, under review. 